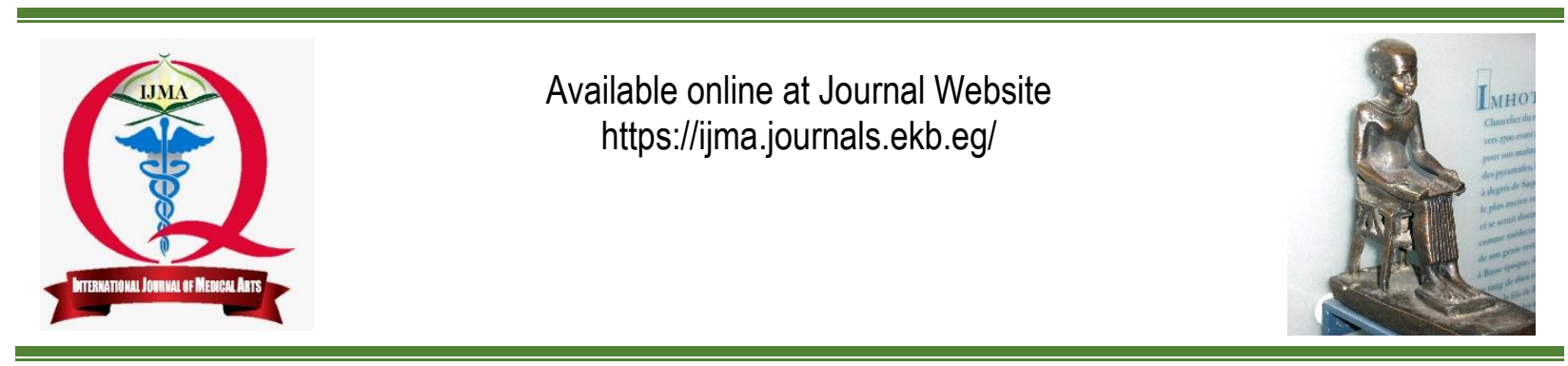

Original article

\title{
Evaluation of the Prevalence and Risk of Metabolic Syndrome in Vitiligo Patients
}

\section{Eman Ahmed Rasheda; Ibrahim Foudab; Emad Elgmal ${ }^{\mathbf{b}}$}

Department of Dermatology, Alexandria University, Egypt [a]

Department of Dermatology, Damietta Faculty of Medicine, Al-Azhar University, Egypt [b]

Corresponding author: Eman Ahmed Rashed

Email: dr.eman.rashed89@gmail.com

Received at: June 11, 2019; Revised at: July 14, 2019;

Accepted at: August 21, 2019; Available online at: August 21, 2019

DOI: 10.21608/ijma.2019.13576.1011

\section{ABSTRACT}

Background: Vitiligo is a common disease of the skin, affecting epidermis and hair follicles. Both genders are affected. The main pathogenic mechanism of the disease is the destruction or inhibition of the epidermal melanocytes which is manifested clinically by presence of variable sized areas of skin depigmentation that can affect any site of the body.

Aim of the work: To estimate the prevalence and risk of metabolic syndrome in vitiligo patients.

Patients and Methods: This is a case control study that was conducted on 90 patients with vitiligo recruited from outpatient clinic, dermatology department of Al Azhar University Hospital in Damietta, and 60 healthy controls. Full history was taken from all the subjects included in the study. The cases were also subjected to careful general examination and dermatological examination to determine the site and the type of the lesion and the presence of any dermatological diseases, laboratory investigations including HDL, TG and FBS were done.

Results: In this study we found that $35.6 \%$ of cases have metabolic syndrome while $33.3 \%$ of healthy controls have metabolic syndrome. We also found that there is no relation between presence of metabolic syndrome and disease extent, progression or stage.

Conclusion: Incidence of metabolic syndrome is slightly higher in cases with vitiligo as compared to the control group. Presence of metabolic syndrome is not associated with disease severity, extent or stage.

Keywords: Vitiligo; Segmental vitiligo; Interleukin; Cytokines; Metabolic Syndrome.

This is an open access article under the Creative Commons license [CC BY] [https://creativecommons.org/licenses/by/2.0/]

Please cite this article as: Rashed EA, Fouda I, Elgamal E. Evaluation of the prevalence and risk of metabolic syndrome in vitiligo patients. IJMA 2019; 1[2]:91-97. 


\section{INTRODUCTION}

Vitiligo is acquired, non-contagious skin disease manifested clinically by presence of progressive depigmented patches in the skin, overlying hair, and oral mucosa. The main mechanism responsible for this disorder is most probably autoimmune leading to destruction or inhibition of melanocytes in the affected skin areas. These depigmented patches wax and wane, but in most cases they are merged together up to involvement of the whole body [1]. The depigmented skin lesions could be traumatic on involvement of certain areas like the face, hands, and genitals especially when affect people from racial clusters with dark skin. Although pathogenic and genetic causes of vitiligo are not fully discovered, the disease has been well-known all over the world for thousands of years because of its prominent appearance 22]. Among disorders of skin pigmentation, vitiligo is the most common disease with prevalence of $0.1-2 \%$ in different populations. The familial predominance is not uncommon; patients suffer from vitiligo had about $20 \%$ positive history of first-degree relatives. The pattern of inheritance suggested to be non-Mendelian. Thus, a multi-factorial genetic cause is proposed[2].

Vitiligo usually presents in different forms: segmental and non- segmental vitiligo[3]. The exact cause of vitiligo is still unknown, however the autoimmune and oxidative stress mechanisms are the two most accepted theories that are responsible for the occurrence of the disease [4]. Many factors including genetic, immunological, neuronal and inflammatory are involved in the pathogenesis of the disease and they are largely responsible for the variation of the systemic manifestations of the disease. The autoimmune response of the body against vitiligo could lead to development of other associated comorbid autoimmune conditions. Moreover, the inflammatory factors and the proinflammatory cytokines are involved in the pathogenesis of vitiligo have been associated with major metabolic complications such as insulin resistance and atherosclerosis [5]. There are several vitiligo scores which are Vitiligo Disease Activity Score [VIDA], Vitiligo European Task Force [VETF], Vitiligo Area Severity Index [VASI], and Vitiligo Extent Tensity Index [VETI] score [6].

The metabolic syndrome [MS] diagnostic criteria include waist circumference more than or equal to
$102 \mathrm{~cm}$ for men and more than or equal to $88 \mathrm{~cm}$ for women, triglycerides more than or equal to $150 \mathrm{mg} / \mathrm{dL}$ or on treatment, HDL less than $40 \mathrm{mg} / \mathrm{dL}$ for men and less than $50 \mathrm{mg} / \mathrm{dL}$ for women or on treatment, blood pressure greater than or equal to $130 / 85 \mathrm{mmHg}$ or on treatment, and glycemic index greater than or equal to $100 \mathrm{mg} / \mathrm{dL}$ or on treatment [7]. It is widely accepted that one of the main mechanisms for the development of MS is the oxidative stress. Melanocytes are cells that mainly located in the skin and have also been identified in other tissues like the adipose tissue. These cells are thought to have anti-oxidant effect through its ability for scavenger of oxygen free radicles and reactive oxygen species [ROS] [7].

In patients with vitiligo there is significant reduction in the melanocytes number and in the process of melanogenesis in the adipose tissue which are suggested as causes for development of MS in those patients [7].

\section{AIM OF THE WORK}

The aim of this study is to evaluate the prevalence and risk of metabolic syndrome in vitiligo patients.

\section{PATIENTS AND METHODS}

Patients: This is a case control study conducted in Al Azhar University Hospital in Damietta and recruited 90 patients with vitiligo and 60 age- and gender matched healthy controls. Informed consent was taken from all patients and controls. The patients were selected according to the following inclusion and exclusion criteria:

Inclusion criteria: Age $>18$ years, affection of $>10 \%$ surface area of the body with vitiligo and no use of local or systemic treatment for the last 3 months before the beginning of the study.

Exclusion criteria: Pregnant and lactating females, type $1 \mathrm{DM}$, secondary hypertension and patients with other dermatological diseases.

Methods: All included participants were subjected to full history taking about the current disease [its onset, course, duration, associated precipitating factors or diseases and results of medical treatment]. In addition, complete general examination was done in a systematic pattern. Blood pressure and waist circumference [WC] were measured. In addition, dermatological examination was performed for all patients with emphasis to 
determine the site and size of the lesion and the presence of any other dermatological disease. Assessment of disease severity was accomplished according to Vitiligo European Task Force [VETF][8]. Then, a venous blood sample was drawn in a sterile collecting tube with gel after $12-h$ fasting. The sample was centrifuged for 15 minutes at $4000 \mathrm{G}$. Serum was obtained and values of HDL cholesterol, triglycerides and fasting blood glucose were determined.

Statistical analysis of the data: Data was analyzed by using statistical package for social science [SPSS] software, version 20 [IBMßSPSS $\AA$, USA, Chicago, IL]. Quantitative data were tested for normality by using the Kolmogorov-Smirnov test and expressed as means with standard deviation or medians with ranges according to data distribution. Categorical data were presented as number and percentages. $P<0.05$ is considered significant. Groups were compared by Chi-Square test, Student [t] test and Mann Whitney tests.

\section{RESULTS}

Table [1] Showed comparison between study and control groups regarding demographic data, risk factors lipid profile and incidence of metabolic syndrome. Both study and control groups were comparable as regard to patient gender, hypertension, lipid profile and fasting blood sugar.
However, cases were significantly younger than controls [43 vs 49 years respectively]. In addition, mean diastolic blood pressure was significantly lower in cases when compared to controls [70 vs $80 \mathrm{mmHg}$ respectively]. Finally, metabolic syndrome was reported in $35.6 \%$ of the study group and in $33.3 \%$ of the control group with no significant difference.

The duration of disease among study group patients, ranged from 1 to 50 months; the mean value was 18.34 months and median value was 17.50 months; $35.6 \%$ of patients had duration $\leq 10$ months; only $11.1 \%$ had duration $>30.0$ months [table 2]. The majority of patients were in the stage I [73.3\%], while $24.4 \%$ were stage II and $2.2 \%$ were in stage III. The disease was progressive in $53.3 \%$ and disease extent ranged from 12.0 to $91.0 \%$ of total body surface area [BSA], the median extent was $22.5 \%$ and mean extent was $25.68 \%$ [Table 3].

When comparing metabolic to non-metabolic syndrome in study group, both were comparable as regard to disease extent, disease stage and patient gender. However, metabolic syndrome was significantly prevalent in non-progressive disease [table 4]. Obese and non-obese subjects in study group were comparable as regard to disease progression, disease stage and extent [table 5].

Table [1]: Comparison between the two study groups

\begin{tabular}{|c|c|c|c|c|c|c|c|}
\hline & & \multicolumn{2}{|c|}{ Cases [n=90] } & \multicolumn{2}{|c|}{ Control $[n=60]$} & \multirow[t]{2}{*}{ Test of sig. } & \multirow[t]{2}{*}{$p$} \\
\hline & & No. & $\%$ & No. & $\%$ & & \\
\hline \multirow[t]{2}{*}{ Sex } & Male & 48 & 53.3 & 32 & 53.3 & \multirow[t]{2}{*}{$\chi^{2}=0.0$} & \multirow[t]{2}{*}{1.000} \\
\hline & Females & 42 & 46.7 & 28 & 46.7 & & \\
\hline \multicolumn{2}{|c|}{ Age [years] } & \multicolumn{2}{|c|}{$43[18-76]$} & \multicolumn{2}{|c|}{$49[20-78]$} & $t=2.270^{*}$ & $0.025^{\star}$ \\
\hline \multirow[t]{4}{*}{ Hypertension } & Normal & 65 & 72.2 & 36 & 60.0 & \multirow{2}{*}{$\chi 2=2.445$} & \multirow[t]{2}{*}{0.118} \\
\hline & Hypertensive & 25 & 27.8 & 24 & 40.0 & & \\
\hline & SBP & \multicolumn{2}{|c|}{$110[90-160]$} & \multicolumn{2}{|c|}{120 [90-150] } & $t=1.660$ & 0.099 \\
\hline & DBP & \multicolumn{2}{|c|}{$70[60-110]$} & \multicolumn{2}{|c|}{$80[60-110]$} & $t=2.248^{*}$ & $0.026^{*}$ \\
\hline \multirow[t]{2}{*}{$\begin{array}{l}\text { Obesity and waist } \\
\text { circumference }\end{array}$} & Non obese Obese & $\begin{array}{l}59 \\
31\end{array}$ & $\begin{array}{l}65.6 \\
34.4\end{array}$ & $\begin{array}{l}37 \\
23\end{array}$ & $\begin{array}{l}61.7 \\
38.3\end{array}$ & $\chi^{2}=0.236$ & 0.627 \\
\hline & WC & \multicolumn{2}{|c|}{$87[67-147]$} & \multicolumn{2}{|c|}{$79.5[65-143]$} & $t=0.643$ & 0.521 \\
\hline \multirow[t]{2}{*}{ HDL } & $\begin{array}{l}\text { Normal } \\
\text { Low }\end{array}$ & $\begin{array}{l}53 \\
37\end{array}$ & $\begin{array}{l}58.9 \\
41.1\end{array}$ & $\begin{array}{l}37 \\
23\end{array}$ & $\begin{array}{l}61.7 \\
38.3\end{array}$ & $\chi^{2}=0.116$ & 0.734 \\
\hline & Min. - Max. & \multicolumn{2}{|c|}{$50[31-82]$} & \multicolumn{2}{|c|}{$50[30-71]$} & $t=1.197$ & 0.233 \\
\hline \multirow[t]{3}{*}{ TG } & Normal & 41 & 45.6 & 32 & 53.3 & \multirow{2}{*}{$\chi 2=0.872$} & \multirow{2}{*}{0.350} \\
\hline & High & 46 & 54.4 & 28 & 46.7 & & \\
\hline & Min. - Max. & \multicolumn{2}{|c|}{$158[49-456]$} & \multicolumn{2}{|c|}{$127[67-345]$} & $\mathrm{U}=2473.50$ & 0.385 \\
\hline \multirow[t]{2}{*}{ FBS } & $\begin{array}{l}\text { Non-Diabetic } \\
\text { Diabetic }\end{array}$ & $\begin{array}{l}55 \\
35\end{array}$ & $\begin{array}{l}61.1 \\
38.9\end{array}$ & $\begin{array}{l}41 \\
19\end{array}$ & $\begin{array}{l}68.3 \\
31.7\end{array}$ & $\chi 2=0.815$ & 0.367 \\
\hline & Min. - Max. & \multicolumn{2}{|c|}{$95[65-404]$} & \multicolumn{2}{|c|}{90 [66 - 373] } & $U=2524.0$ & 0.499 \\
\hline No met & yndrome & 58 & 64.4 & 40 & 66.7 & $\chi 2=0.078$ & 0.779 \\
\hline Metab & drome & 32 & 35.6 & 20 & 33.3 & & \\
\hline
\end{tabular}

$\chi 2$ :Chi square test t: Student t-test $U$ : Mann-Whitney U test; $p: p$ value for comparing between the two studied groups; *: Statistically significant at $p \leq 0.05$ 
International Journal of Medical Arts.

Table [2]: Distribution of the studied cases according to duration of disease [n=90]

\begin{tabular}{|c|c|c|}
\hline Duration of disease [months] & No. & $\%$ \\
\hline$\leq 10$ & 32 & 35.6 \\
\hline$>10-20$ & 22 & 24.4 \\
\hline$>20-30$ & 26 & 28.9 \\
\hline$>30 \quad$ Min. - Max.; Mean \pm SD.; Median & 10 & 11.1 \\
\hline \multicolumn{2}{|c|}{$1.0-50.0 ; 18.34 \pm 12.71 ; 17.50$} \\
\hline
\end{tabular}

Table [3]: Descriptive analysis of the studied cases according to progression, stage and extent [\%] $[\mathrm{n}=90$

\begin{tabular}{|c|c|c|}
\hline \multicolumn{1}{|c|}{} & No. & $\%$ \\
\hline Stage & & 73.3 \\
\hline I & 66 & 24.4 \\
\hline III & 22 & 2.2 \\
\hline Progression & 2 & 46.7 \\
\hline Negative & 42 & 53.3 \\
\hline Positive & 48 & \\
\hline Extent [\%] & \multicolumn{2}{|c|}{} \\
\hline Min.-Max.; Mean \pm SD.; Median & $12.0-91.0 ; 25.68 \pm 11.85 ; 22.50$ \\
\hline
\end{tabular}

Table [4]: comparison between MS positive and MS negative patients in case group [ $\mathrm{n}=90]$

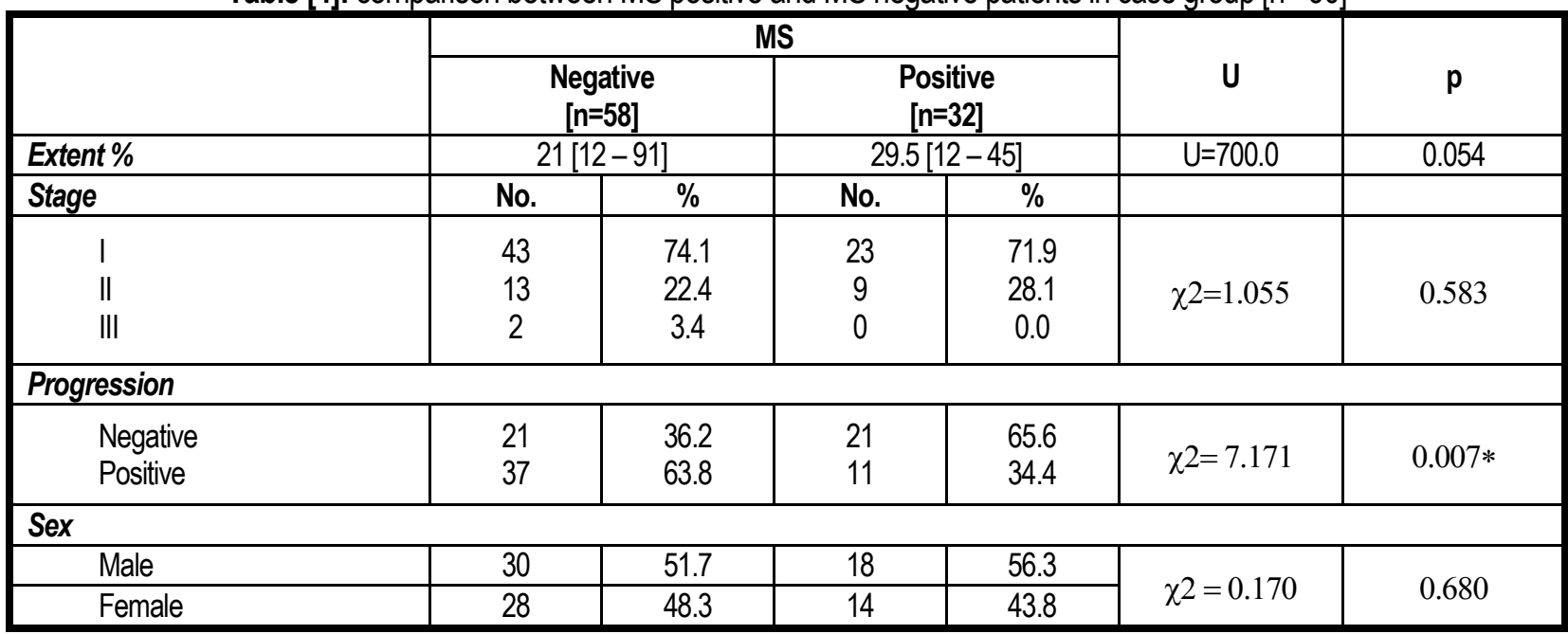

$\chi 2$ :Chi square test U: Mann-Whitney U test; $p: p$ value for comparing between MS positive and MS negative cases; *: Statistically significant at $\mathrm{p} \leq 0.05$

Table [5]: Relation between waist circumference and different parameters in case group [ $\mathrm{n}=90]$.

\begin{tabular}{|c|c|c|c|c|c|c|}
\hline & \multicolumn{4}{|c|}{ Waist circumference } & \multirow{3}{*}{ Test of sig } & \multirow{3}{*}{$p$} \\
\hline & \multicolumn{2}{|c|}{ Non obese [n= 59] } & \multicolumn{2}{|c|}{ Obese [n= 31] } & & \\
\hline & No. & $\%$ & No. & $\%$ & & \\
\hline $\begin{array}{c}\text { Progression } \\
\text { Negative } \\
\text { Positive }\end{array}$ & $\begin{array}{l}26 \\
33 \\
\end{array}$ & $\begin{array}{l}44.1 \\
55.9 \\
\end{array}$ & $\begin{array}{l}16 \\
15 \\
\end{array}$ & $\begin{array}{l}51.6 \\
48.4 \\
\end{array}$ & $\chi 2=0.465$ & 0.495 \\
\hline \multicolumn{7}{|l|}{ Stage } \\
\hline I & 40 & 67.8 & 26 & 83.9 & \multirow{3}{*}{$\chi^{2}=2.623$} & \multirow{3}{*}{0.262} \\
\hline$\|$ & 17 & 28.8 & 5 & 16.1 & & \\
\hline III & 2 & 3.4 & 0 & 0.0 & & \\
\hline \multicolumn{7}{|l|}{ Extent \% } \\
\hline Min. - Max. & \multicolumn{2}{|c|}{$12.0-91.0$} & \multicolumn{2}{|c|}{$12.0-45.0$} & \multirow{3}{*}{$U=909.0$} & \multirow{3}{*}{0.963} \\
\hline Mean \pm SD. & \multicolumn{2}{|c|}{$25.97 \pm 12.61$} & \multicolumn{2}{|c|}{$25.13 \pm 10.42$} & & \\
\hline Median & \multicolumn{2}{|c|}{22.0} & \multicolumn{2}{|c|}{23.0} & & \\
\hline
\end{tabular}

$\chi 2:$ Chi square test MC: Monte Carlo U: Mann Whitney test; $p$ : $p$ value for association between waist circumference and different parameters 


\section{DISCUSSION}

Although vitiligo is known for thousands of years, but the exact pathogenic mechanism of the disease is still unknown. Many mechanisms such as autoimmunity, genetic factors and involvement of inflammatory cytokines have been suggested to be responsible for the start of the disease [10]. Many other factors are suggested to be involved in the process of disease mechanism including melanocyte disturbances and destruction, presence of antibodies against melanin-concentrating hormone $[\mathrm{MCH}]$ receptor, the increased production of $\mathrm{MCH}$, increased homocysteine levels, high levels of catecholamine, reactive oxygen species, stress and cytomegalovirus [11]. Metabolic syndrome is a serious condition that includes abdominal obesity, increased levels of fasting blood sugar [hyperglycemia], dyslipidemia and hypertension [12]

Vitiligo is a syndrome with systemic manifestations and not confined only to the skin, so metabolic disturbances are common findings in patients with vitiligo. On comparison between girls with vitiligo and healthy controls, the former showed reduced values of $\mathrm{HDL}$ cholesterol and hgih triglyceride levels [13]. Furthermore, the incidence of vitiligo is increased in many diabetic patients as reported by many studies [14].

Autoimmunity and oxidative stress could initiate many inflammatory and immunological cascades that are responsible for systemic manifestations and as well as skin involvement in cases with vitiligo [5]. Melanin pigment has been identified in the adipose tissue where it is suggested to have both anti-inflammatory and antioxidant effects ${ }^{[15]}$. A reduced number of melanocytes and defective melanogenesis in the adipose tissue could decrease the anti-inflammatory effects of the melanocytes, and causes overproduction and accumulation of the oxygen free radicals in vitiligo, with subsequent development of metabolic syndrome. Moreover, many mechanisms have been proposed to be responsible in metabolic syndrome pathogenesis in vitiligo, such as insulin resistance, disturbed lipids and other diseases of metabolism, due to increased inflammatory cytokines and autoimmunity of melanocytes [16].

In our study, it was found that [35.6\%] of cases has metabolic syndrome while [33.3\%] of control group has metabolic syndrome. This is in agreement with Ataş and Gönü|[7] who revealed that the presence of metabolic syndrome was higher in the cases of vitiligo [38.1\%] than in the controls [21.5\%]. Also, Sharma et al.[6] found that $24 \%$ of cases have metabolic syndrome and $12 \%$ of control group have metabolic syndrome

In previous literature, studies searching the link between vitiligo and metabolic syndrome yielding controversial results. For example Karadag et al.[17] have advocated such link, these link was contradicted by Pietrzak et al.[5] who reported that vitiligo patients had a better lipid profile in comparison with the control group.

In our study, mean age for cases was $40.87 \pm$ 14.26 and for controls it was $46.38 \pm 15.06$. While Sharma et al. [6] did their study with mean age $43.5 \pm 10.5$ for cases and $42.3 \pm 11.5$ for controls.

The lower mean age of our study participants is due to exclusion of patients aged lower than 30 years in Sharma et al. ${ }^{[6]}$ study.

In the current study, male percent was 53.3 while female percent was 46.7 for both cases and control groups. While Sharma et al. [i] did their study on $66 \%$ males and $34 \%$ females for cases and $64 \%$ males and $36 \%$ females for control groups. Male dominance in Sharma et al. [6] study may be due to the cultural conservation among Indian females who report for treatment mostly when vitilgo is located on the cosmetically important areas. We also found that there is no relation between presence of metabolic syndrome and disease extent, progression or stage. Similar results were reported in Sharma et al. [6], study.

About diastolic BP, in our study, the median was 70 in case group and 80 in control group, while Ataş and Gönü|[7] found that it was 70 in both groups. Also, Sharma et al. ${ }^{[6]}$ found that mean of diastolic BP was $78.42 \pm 6.86$ in cases and $78.32 \pm 6.70$ in control group.

Our study revealed that $27.8 \%$ of cases and $40 \%$ of control group have systolic BP $>130$ or diastolic BP >85. On the other hand, Sharma et al. [6] found it was $28 \%$ in cases and $40 \%$ in control group.

In our study, median of waist circumference was higher in cases than control group. It was 87 in case group and 79.5 in control group. In contrast to our 
study, Ataş and Gönü[[7] found it was 93 in case group and 96 in control group. Also, Sharma et al. [6] found that mean of waist circumference was $81 \pm 10.9$ for cases and was $81.82 \pm 8.41$ for controls.

Our study revealed that $34.4 \%$ of cases and 38.4 $\%$ of control have increased waist circumference. On the other hand Sharma et al. [6] found that 37\% of cases and $31 \%$ of control group have higher waist circumference.

In our study we found that $38.9 \%$ of cases have FBS $>100 \mathrm{mg} / \mathrm{dl}$ and $31.7 \%$ of controls have FBS $>100$. Also Sharma et al. ${ }^{[6]}$ found FBS was $>100$ in $25 \%$ of cases and $16 \%$ of control group. Insulin resistance is considered as the cornerstone in the pathogenesis of MS AND within the last decades, many studies have revealed the relation between proinflammatory cytokines such as TNFa, IL1 and IL6 and the development of insulin resistance-the. Furthermore, these inflammatory markers were found to be elevated in patients with vitiligo.

In our study, median of TG was 158 for cases and 127 for control group. This is in agreement with Ataş and Gönü|[7] that found it was 140 in cases and 130in control group. Also Sharma et al. ${ }^{[6]}$ found that mean of TG was $144.54 \pm 47.61$ in cases and $133.34 \pm 29.85$ in control group.

In our study, percent of cases having TG $>150$ $\mathrm{mg} / \mathrm{dl}$ was 54.4 and percent of controls was 46.7 . This is in agreement with Sharma et al. [6] that found it was 41 for cases and 24 for controls.

Our study revealed that median of HDL was 50 for both case group and control group. While Ataş and Gönül[7] found it was 48 in case group and 46 in control group. While Sharma et al. [6] found mean of $\mathrm{HDL}$ was $42.14 \pm 5.92$ for case group and $44.11 \pm 7.68$ for control group. We had $41.1 \%$ of cases and $38.3 \%$ of control having low HDL. This is in agreement with Sharma et al. ${ }^{[6]}$ that found the percent was 58 in case group and 53 in control group.

Karadag et al. [17] found that, in vitiligo patients, HDL-cholesterol was significantly decreased and the LDL/HDL ratio was increased. The same authors also found that even in non-diabetic patients with vitiligo, a higher insulin resistance and C-peptide values were observed when compared to control group. These data confirm the association between metabolic syndrome and vitiligo.
Comparable results were reported in an earlier study done by Pietrzak et al. [18]

More recently, adipose tissue, especially the white adipose tissue, was considered as an endocrine tissue and the bioactive substances secreted from adipose tissues such as, adipokines [like leptin, adiponectin, resistin, vaspin, visfatin] as well as TNFa, IL-6, MCP-1-were shown to affect immunity, glucose and lipid metabolism. Any abnormalities in the production and secretion of these biomarkers could be responsible for the occurrence of MS. Reduction in number of melanocytes in the adipose tissue leads to decreased melanogenesis with subsequent predisposition to inflammation and increase in ROS, lipid peroxidation, protein oxidation/ oxidative DNA damage, etc. enhancement of lipid peroxidation in the epidermis and the adipose tissue could be considered as the cause of lipid abnormalities as well as the increased prevalence of metabolic syndrome, as also seen in our cases.

In conclusion, incidence of metabolic syndrome is slightly higher in vitiligo patients than in control group. Presence of metabolic syndrome is not associated with disease severity, extent or stage.

\section{Conflict of Interest}

Authors declare that, there was no conflicts of interest.

\section{REFERENCES}

1. Ezzedine K, Lim H, Suzuki T, Katayama I, Hamzavi I, Lan C, Goh B, Anbar T, et al. Revised classification/nomenclature of vitiligo and related issues: the Vitiligo Global Issues Consensus Conference. Pigment Cell Melanoma Res. 2012; 25[3]: E1-E13. [DOI:10.1111/j.1755-148X.2012. 00997.x].

2. Alikhan A, Felsten LM, Daly M, Petronic-Rosic V. Vitiligo: a comprehensive overview: part I. Introduction, epidemiology, quality of life, diagnosis, differential diagnosis, associations, histopathology, etiology, and work-up. J Am Acad Dermatol. 2011; 65 [3]: 473-491.[DOI:10.1016/j.jaad.2010.11.061].

3. Craiglow BG, King BA. Tofacitinib citrate for the treatment of vitiligo: a pathogenesis-directed therapy. JAMA Dermatol. 2015;151[10]:1110-2. [DOI: 10.1001/jamadermatol.2015.1520].

4. Xie H, Zhou F, Liu L, Zhu G, Li Q, Li C, Gao T. Vitiligo: how do oxidative stress-induced autoantigens trigger autoimmunity? J Dermatol Sci. 2016; 81[1]: 3-9. [DOI:10.1016/j.jdermsci. 2015.09. 003].

5. Pietrzak A, Bartosińska J, Hercogová J, Lotti TM, Chodorowska G. Metabolic syndrome in vitiligo. Dermatol Ther. 2012;25 (Suppl 1):S41-S3. [DOI:10.1111/dth.12012]. 
6. Sharma Y, Bansal P, Menon S, Prakash N. Metabolic syndrome in vitiligo patients among a semiurban Maharashtrian population: A case control study. Diabetes Metab Syndr. 2017;11(Suppl 1):S77S80.[DOI:10.1016/j.dsx.2016.12.009].

7. Ataş $\mathbf{H}$, Gönül $\mathbf{M}$. Increased risk of metabolic syndrome in patients with vitiligo. Balkan Med J. 2017; 34 [3]: 219. [DOI:10.4274/balkanmedj. 2016. 1005].

8. Hamzavi I, Jain H, McLean D, Shapiro J, Zeng H, Lui H. Parametric modeling of narrowband UV-B phototherapy for vitiligo using a novel quantitative tool: the Vitiligo Area Scoring Index. Arch Dermatol. 2004;140[6]:677-83. [DOI:10.1001/archderm. 140.6. $677]$.

9. Wong PC, Leung Y-Y, Li EK, Tam L-S. Measuring disease activity in psoriatic arthritis. Int J Rheumatol. 2012; 2012: 839425. [DOI:10.1155/2012/839425].

10. Kumar S, Nayak CS, Padhi T, Rao G, Rao A, Sharma V, Srinivas C. Epidemiological pattern of psoriasis, vitiligo and atopic dermatitis in India: Hospital-based point prevalence. Indian Dermatol Online J. 2014;5[Suppl 1]:S6-8.[DOI:10.4103/22295178. 144499].

11. Coelho M, Oliveira T, Fernandes R. Biochemistry of adipose tissue: an endocrine organ. Arch Med Sci: 2013;9[2]:191-200.[DOI:10.5114/aoms.2013.33181].

12. Moretti S, Spallanzani A, Amato L, Hautmann G, Gallerani I, Fabiani M, Fabbri P. New insights into the pathogenesis of vitiligo: imbalance of epidermal cytokines at sites of lesions. Pigment Cell Res. 2002; 15[2]:87-92.[PMID:11936274].
13. Lv Y, Li Q, Wang L, Gao T. Use of anti-tumor necrosis factor agents: a possible therapy for vitiligo. Medical hypotheses. 2009;72[5]:546-7. [DOI: 10.1016/ j.mehy.2008.12.033].

14. Page S, Chandhoke V, Baranova A. Melanin and melanogenesis in adipose tissue: possible mechanisms for abating oxidative stress and inflammation? obesity Rev. 2011;12[5]:e21e31.[DOI:10.1111/j.1467-789X.2010.00773.x].

15. Karadag A, Tutal E, Ertugrul D, Akin K, Bilgili S. Serum holotranscobalamine, vitamin B12, folic acid and homocysteine levels in patients with vitiligo. Clin Exp Dermatol. 2012;37[1]:62-4.[DOI:10.1111/j.13652230.2011.04142.x].

16. Yudkin JS, Stehouwer C, Emeis J, Coppack S. Creactive protein in healthy subjects: associations with obesity, insulin resistance, and endothelial dysfunction: a potential role for cytokines originating from adipose tissue? Arterioscler Thromb Vasc Biol.y. 1999;19[4]:972-8. [DOI:10.1161/01. atv. 19.4. 972].

17. Karadag AS, Tutal E, ErtugruI DT. Insulin resistance is increased in patients with vitiligo. Acta Derm Venereol. 2011: 91: 541-544. [DOI:10.2340/ 00015555-1141].

18. Pietrzak A, Lecewicz-Torun' B, Urban J. Comparison of serum lipid in girls affected with vitiligo and control group. Ann Univ Mariae Curie Sklodowska Med. 2000: 55: 269-274. [PMID: 11482085] 